\title{
Editorial
}

\section{Gregor Prinzensing}

Online publiziert: 23. November 2015

(C) Springer-Verlag Berlin Heidelberg 2015

\section{Liebe Leserinnen und Leser,}

mit dem letzten Heft dieses 73. Jahrgangs von „Raumforschung und Raumordnung“ präsentieren wir Ihnen ein Varia-Heft ausschließlich mit wissenschaftlichen Beiträgen: aus der raumbezogenen Forschung zur Energiewende, aus der Demographieforschung und aus der Stadtentwicklungsund Mobilitätsforschung.

Den Anfang macht jedoch ein englischsprachiger wissenschaftlicher Beitrag mit einem methodisch ausgerichteten Vorschlag. Jürgen Friedrichs und Jörg Blasius stellen Ihnen hier „The Dwelling Panel - A New Research Method for Studying Urban Change“ vor. Während klassische sozialwissenschaftliche Panel-Studien sich auf Haushalte bzw. Personen in Haushalten beziehen, schlagen die Autoren hier eine andere Untersuchungseinheit vor. Mit den bisherigen Methoden lassen sich nämlich gebietsbezogene Veränderungsprozesse etwa innerhalb von Nachbarschaften kaum über längere Zeit studieren bzw. überhaupt angemessen erfassen. Als Bezugsgrößen für die hier vorgeschlagene Art von Panel-Studien sollen deshalb konkrete Wohnstätten (ein Sample von verschiedenen Wohnungen oder auch Einfamilienhäusern innerhalb eines Gebietes) ausgewählt werden. Damit lassen sich nach Ansicht der Autoren kleinräumliche Veränderungsprozesse wie die Gentrifizierung oder der soziale Abstieg eines Quartiers oder auch Veränderungen im Abstimmungsverhalten von Wählern innerhalb eines Wahlkreises besser untersuchen. Die Autoren beschreiben die

G. Prinzensing $(\bowtie)$

Akademie für Raumforschung und Landesplanung (ARL), Leibniz-Forum für Raumwissenschaften,

Hohenzollernstraße 11,

30161 Hanover, Deutschland

E-Mail: prinzensing@arl-net.de
Methode und diskutieren ihre Erfordernisse sowie spezifische Vorteile dieser Herangehensweise unter anderem auch am Beispiel der von ihnen durchgeführten Kölner Wohnstättenstudie (das „Cologne Dwelling Panel“). Hier wurden 2010 und 2011 in aufeinander folgenden Befragungswellen für zwei benachbarte Kölner Quartiere vergleichend Befunde erhoben. Im Vergleich mit klassischen Panel-Studien können hier nach mehreren Befragungswellen auch die Ausfälle (wenn Interviewpersonen nicht erreichbar oder nicht mehr zur Mitwirkung bereit waren) in die Auswertung einbezogen und als Indiz von Veränderungen räumlich interpretiert werden.

Der anschließende Beitrag von Jörg Radtke widmet sich dem „Zusammenspiel von Raum und Technik bei der Etablierung Erneuerbarer Energien“. Er untersucht die Auswirkungen der Energiewende und die damit verbundene Transformation von Räumen und vergleicht dies mit den Raum strukturierenden Effekten, wie sie im Rahmen konventioneller Energieerzeugung auftreten. Dazu greift er unter anderem Überlegungen von Bruno Latour auf (vor allem dessen Forderung nach dem „Einbezug der Dinge“) und integriert hier auch Michel Foucaults Konzept ,anderer Räume“. In Betracht genommen werden dabei Fragen der Abgrenzung von Technikräumen gegenüber der Umwelt, ebenso umgekehrt Prozesse der Hybridisierung (der Verschmelzung von Natur, Technik und Gesellschaft zu einem Konglomerat) sowie schließlich die dabei sich ergebenden Gestaltungs- und Partizipationsmöglichkeiten in lokalen Kontexten. Die gewonnenen Einsichten werden auf Fallbeispiele aus den Bereichen Windenergienutzung und den Ausbau der Stromnetze bezogen. Der Beitrag schließt mit einem Plädoyer für gut austarierte Raumgestaltungsprozesse bei der Umsetzung der Energiewende. Situativ angemessene Lösungen (z. B. bei Standortentscheidungen) ließen sich nach Ansicht des Autors dann am ehesten verwirklichen, 
wenn dies im Zusammenwirken von lokaler Bevölkerung und den weiteren Verfahrensbeteiligten geschehe und auf einem offenen und aufgeschlossenen Umgang mit lokalen Besonderheiten beruhe.

Mit der Frage, welche demographischen Effekte sich künftig möglicherweise regional unter Fortschreibung der gegenwärtigen Alterungs- und Schrumpfungsbedingungen zeigen, befasst sich der Beitrag „Auswirkungen des demographischen Wandels auf die regionale Bevölkerungsdynamik in Deutschland“" von Frank Swiaczny. Der Autor legt seinen über den bisherigen Prognosezeitraum bis 2030 hinausreichenden Überlegungen zu veränderten Wohn- bzw. Wanderungspräferenzen die Daten und Modellrechnungen des Statistischen Bundesamtes sowie des Bundesinstituts für Bau-, Stadt- und Raumforschung (BBSR) zugrunde. In einer alternativen Modellrechnung geht er von der Annahme aus, dass in Zukunft die Prozesse der Binnenwanderung in Verbindung mit der räumlichen Verteilung des künftigen Zuwachses im Wohnungsbestand anderen Mustern folgen werden als in der Vergangenheit. Der Beitrag kommt zum Ergebnis, dass die bisherigen Bevölkerungsprognosen bis in die 2030er-Jahre einen Trend suggerieren, der die langfristige Bevölkerungsentwicklung in suburbanen und ländlichen Regionen zu positiv zeichnet. Nach Ansicht des Autors werden in Zukunft die Schwächen der durch Bevölkerungsrückgang und Abwanderung benachteiligten Standorte in der Konkurrenz um Wanderungsgewinne deutlicher zutage treten als bisher.

Der vierte Beitrag von Mathias Wilde, Andreas Blechschmidt und Martin Lanzendorf diskutiert die grundlegenden Potenziale und Grenzen kompakter Stadtentwicklung und untersucht am Beispiel der Planungspraxis der Stadt
Leipzig, wie „Integrierte Stadtentwicklung und die Gestaltung nachhaltiger Mobilität" zusammengebracht werden können. Eine Stadtentwicklung, die auf nachhaltige Mobilität und einen zukunftsfähigen Stadtverkehr zielt, sollte nach Ansicht der Autoren nicht nur infrastrukturbezogene Konzepte etwa zur Förderung des Wohnens im Innenbereich, zur Stärkung der städtischen Zentren oder zur Förderung der Nahmobilität in Betracht ziehen. Wichtig sind darüber hinaus auch Verbesserungen bei den übergeordneten Rahmenbedingungen (z. B. Konzepte zur Stärkung der Zusammenarbeit innerhalb und zwischen Kommunen sowie die Entwicklung integrierter städtischer Mobilitätskonzepte) und die damit zusammenhängenden politischen, kommunikativen und organisatorischen Maßnahmen. Die Autoren empfehlen hier unter anderem eine an Nachhaltigkeitszielen orientierte Gestaltung rechtlicher und politischer Rahmenbedingungen sowie insgesamt eine stärkere Berücksichtigung auch regionaler Verknüpfungen in städtischen Planungen. Für eine wirksame Umsetzung liegt es ihrer Ansicht nach nahe, diese Fragen im Kontext der, ,integrierten Stadtentwicklung" zu diskutieren und dies auch zur Fortentwicklung bzw. Qualifizierung dieses Ansatzes zu nutzen.

Zuletzt eine Mitteilung in eigener Sache: Wladimir Sgibnev vom Leibniz-Institut für Länderkunde in Leipzig scheidet zum Jahresende aus dem Team der Schriftleitung für die Zeitschrift aus und eine neue Kollegin, Angelika Krehl vom ILS - Institut für Landes- und Stadtentwicklungsforschung in Dortmund, tritt seine Nachfolge an. An Herrn Sgibnev geht unser herzlicher Dank für die geleistete Unterstützung bei der gemeinsamen Herausgabe der „Raumforschung und Raumordnung" in den letzten zwei Jahren. 